GLUT1 deficiency, a non-invasive initial test, may be preferred to lumbar puncture in children with early-onset absence epilepsy suspected of having GLUT1 deficiency syndrome. (Suls A et al. Ann Neurol 2009;66:415-419). GLUT1 deficiency may underlie a significant proportion $(>10 \%)$ of early-onset absence epilepsies. The ketogenic diet, supplying an alternative fuel to glucose for brain energy metabolism, is an effective treatment for drug refractory seizures associated with GLUT1 deficiency. The transient improvement in attention and occipital alpha rhythm following glucose loading demonstrates the critical dependence of cerebral function and alpha EEG activity on glucose as brain fuel. Of interest, a trial of a chronic hyperglycemic diet failed to provide a protracted improvement in seizure control (Akman CI, De Vivo DC et al. Preliminary observation).

\title{
EXPANDING GENETIC AND CLINICAL SPECTRUM OF GLUT1D
}

A multicenter international genetic and clinical study of GLUT1 deficiency received 132 requests for mutational analysis of SLC2A1 gene from 2004-8. Mutations identified in 57 patients (43\%) were novel in 37 , known in 6 , and multiple exon deletions in 6. Clinical data retrospectively collected by questionnaire revealed three different phenotypes: 1) classical (84\%), early onset (<2 years) $(65 \%)$ and late-onset $(18 \%) ; 2)$ non-classical with mental retardation and movement disorder, without epilepsy (15\%); and 3) one adult case with minimal symptoms. The ketogenic diet controlled epilepsy in $86 \%$ and reduced movement disorders in $48 \%$ patients with classical phenotype and in $71 \%$ of non-classical. Delay in diagnosis of classical cases was 6.6 years (range, 1 month-16 years). CSF glucose was $<2.5 \mathrm{mmol} / 1$ (range $0.9-2.4 \mathrm{mmol} / \mathrm{l}$ ), and CSF:blood glucose ratio was $<0.5$ in all but one (range 0.19-0.52). CSF lactate was low to normal. Type of mutation was related to severity of phenotype. CSF:blood glucose ratio was related to type of mutation and phenotype. (Leen WG, Klepper J, Verbeek MM, et al. Glucose transporter-1 deficiency syndrome: the expanding clinical and genetic spectrum of a treatable disorder. Brain March 2010;133(3):655-670). (Respond: WG Leen MD, Nijmegen Medical Centre 935 Neurology, PO Box 91016500 HB Nijmegen, The Netherlands. E-mail: w.leen@neuro.umcn.nl).

COMMENT. Lumbar puncture with CSF glucose determination was the key to diagnosis of GLUT1 deficiency and prompt treatment with the ketogenic diet. Seizures were not a symptom in $15 \%$ of patients.

\section{NEUROLOGICAL VARIANTS OF LESCH-NYHAN DISEASE}

A prospective, multicenter international study of neurological manifestations of Lesch-Nyhan disease variants is reported from Emory University School of Medicine, Atlanta, GA. Of 46 patients (age range 3 to 65 years) from 34 families, 43 (93\%) had neurological dysfunction. Classic cases with self-injurious behavior were excluded. Variant cases were male and had overproduction of uric acid and deficiency of the enzyme hypoxanthine-guanine phosphoribosyl-transferase or evidence for an HPRT gene mutation. Eight had gout, 6 had kidney problems, and 1 had a tophus. Delay in motor and speech development was common. Motor abnormalities in $42(91 \%)$ ranged from subtle 